\title{
DESIGN AND APPLICATION OF BARCODE DIAMETER-AT-BREAST-HEIGHT TAPE IN FOREST INVENTORIES
}

\author{
ZHou, D. Q. - HE, X. J. ${ }^{*}-$ CHEN, G. W. \\ The Third Surveying and Mapping Institute of Guizhou Province, Guiyang 550004, China \\ (e-mail:214955874@qq.com (Zhou,D.Q.),521010515@qq.com (Chen, G.W.); \\ phone/ fax: +86-1-307-8590-698) \\ ${ }^{*}$ Corresponding author \\ e-mail: 623094714@qq.com; phonel fax: +86-1-307-8590-698 \\ (Received $17^{\text {th }}$ Jun 2019; accepted $2^{\text {nd }}$ Sep 2019)
}

\begin{abstract}
Diameter at breast height (DBH) is one of the most important factors in forest inventories. A diameter tape is a traditional tool for measuring DBHs and is often used to obtain references when testing other DBH tools. However, reading measurements with this tool is subjective and may result in low-accuracy values. In this paper, two types of diameter tapes with barcoding (black \& white tape and multicolour tape) were designed based the traditional diameter tape. A new algorithm was designed and developed as an application that can be installed on a smartphone with a wide-angle camera to decode and store readings of these new tapes. This system was tested in typical forest plots. The results showed that when comparing the mean values measured multiple times with reference values obtained with a traditional tape, the bias was not particularly significant $(1.3 \mathrm{~mm})$. The RMSE of measurements using these new tools $(5.6 \mathrm{~mm})$ was smaller than that obtained using a traditional tool $(9.2 \mathrm{~mm})$. Furthermore, this new approach reduced the subjectivity of different observers reading the data. Therefore, these new tapes may be better alternatives to a traditional tape.
\end{abstract}

Keywords: forest inventory, standing tree, diameter at breast height, smartphone, automatic measurement

\section{Introduction}

Field survey is an important means of gathering information from forests. The information collected usually includes diameter at breast height (DBH), tree height and crown width, which can be used to estimate stock volume and biomass models (Maia et al., 1999; Drexhage and Colin, 2001; Popescu et al., 2003; Ouimet et al., 2008) and to establish remote sensing inversion models (Riaño et al., 2003; Wieser et al., 2017). The accuracy of the collected information directly determines the accuracy of such models. $\mathrm{DBH}$ is easier to measure and more important than other variables. DBH has strong correlations with tree height, stock volume and biomass (Gering and May, 1995; Popescu et al., 2003; Ouimet et al., 2008; Sumida et al., 2013). Therefore, the accuracy, efficiency, and objectivity of DBH measurements are of great interest.

Caliper and diameter tape are traditional measurement tools. These tools are inefficient and yield subjective results because they require contact with the trees and the manual reading of measurements. Some new, non-contact tools, such as relascopes and dendrometers, have been developed and used for DBH measurements. Kalliovirta et al. (2005) tested a laser-relascope, which is a combination of a relascope and a dendrometer, under typical forest conditions. They compared the measurements with the values gathered by a steel caliper and obtained a standard error of $8.2 \mathrm{~mm}$. The accuracy of the laser-relascope measurements depends on distance, tree measurement time, DBH and observer experience. 
Terrestrial laser scanning (TLS) and mobile laser scanning (MLS) are considered alternatives to traditional measurement tools. These devices can be used to scan a sample plot and generate three-dimensional (3-D) point cloud data with high position accuracy. Through filtering, reconstruction and some additional steps, sample plot information including DBH measurements can be obtained from the data (Watt, 2005; Maas et al., 2008; Liang et al., 2012; Yu et al., 2013; Saarinen et al., 2013; Liang et al., 2014a; Srinivasan et al., 2015; Vastaranta et al., 2015; Olofsson and Olsson, 2017). Maas et al. (2008) used single-station and multi-station observations to measure the DBH of trees and compared the results with traditional caliper measurements. They found that the multiple-scan data was superior to the single-scan data, with a root mean squared error (RMSE) of $1.5 \mathrm{~cm}$ vs. an RMSE of $3.2 \mathrm{~cm}$. Liang et al. (2014a) tested an MLS system composed of a high-performance laser scanner, a navigation unit, and a six-wheeled all-terrain vehicle in forest inventories, and the RMSE of the estimations of DBH was $2.36 \mathrm{~cm}$. However, the high expense of the devices and the limited availability of cloud-processing software for the obtained cloud data of sample plots may limit the applications of TLS and MLS for forestry measurements.

A camera is another effective measuring tool that has been widely used in forest inventory (Juujarvi et al., 1998; Melkas et al., 2008; Liang et al., 2014b; Miller et al., 2015; Varjo et al., 2019). The device is typically used in one of two ways (to analyze a single photo attached to some conditions or a point cloud generated from multiple photos). Melkas et al. (2008) tested a laser-camera that combined a digital reflex camera with an integrated laser line generator. Tree diameters measured with the device were compared with measurements conducted with a steel caliper. The standard error of the diameter observations was $6 \mathrm{~mm}(5.3 \%)$, and the proportion of bias was $2.5 \mathrm{~mm}$. However, this method may not be as efficient as the direct use of a diameter tape. Liang et al. (2014b) evaluated a point cloud generated from an uncalibrated hand-held camera and compared with measurements obtained with a steel tape. The RMSE of the DBH estimates of individual trees was $2.39 \mathrm{~cm}$, which means that the results may not be very accurate. The color point cloud generated by this approach can be processed in the same way as a point cloud obtained by TLS or MLS, but the automated image matching procedure is very difficult and introduces more redundant error than does a TLS method.

A smartphone is a device that integrates sensors such as a wide-angle camera, gyro system, and GPS. As a low-cost, portable and developable device, the smartphone has been widely used in various industries including forest inventory (Villasante and Fernandez, 2014; Bijak and Sarzyński, 2015; Vastaranta et al., 2015; Molinier et al., 2016; Qu et al., 2017). Vastaranta et al. (2015) evaluated a related app (TRESTIMATM) in conducting sample plot measurements and included a function to measure stem diameter automatically. The app was used to measure basal area median tree diameter $(\mathrm{DgM})$ in the test. The measurements were compared with values obtained by a caliper. Biases for the DgM measurements varied from $-1.4 \%$ to $3.1 \%$, and the RMSE ranged from $5.2 \%$ to $11.6 \%$ among different tree species. Clearly, these errors are larger than desired.

Although many advanced devices have been used for DBH measurements, a caliper and diameter tape are still considered the most accurate measurement tools, and measurements obtained with these devices have been widely used as true values for comparison tests. However, the observations made with these two instruments and the recording of results are subjective, which will affect the validity of comparative tests. 
To the present authors' knowledge, no tool has yet been developed that measures diameter as accurately as these two traditional tools and that does not yield subjective measurements.

In this study, a new diameter tape (barcode diameter tape) was developed. The scale on this tape was coded with special barcodes to allow measurements to be decoded and recorded using a smartphone with a wide-angle camera. A new algorithm to read and parse images with the barcodes was designed and developed into a smartphone application. This system seeks to obtain measurements of the same level of accuracy as traditional measurement tools and to overcome the subjectivity of observations and records. The new system was tested in typical forest conditions, and the accuracy of the DBH measurements was examined.

\section{Barcode diameter tape}

\section{Structure of the barcode diameter tape}

Diameter tape is a tool for measuring the diameter (D) of a tree at breast height. The measurement principle of conventional diameter tape is to measure the circumference (C) of the tree at breast height. $\mathrm{D}$ is obtained by the formula $\mathrm{D}=\mathrm{C} / \pi$. Thus, a linear relationship can be found between DBH and circumference, wherein each $\pi \mathrm{cm}$ on the diameter tape is equal to $1 \mathrm{~cm} \mathrm{DBH} \mathrm{(Fig.} \mathrm{1).} \mathrm{The} \mathrm{unit} \mathrm{interval} \mathrm{on} \mathrm{the} \mathrm{diameter} \mathrm{tape} \mathrm{is}$ designed for the $\pi \mathrm{cm}$, sub-scale value for $\pi / 10$, and the readout values from the tape are the DBH values.

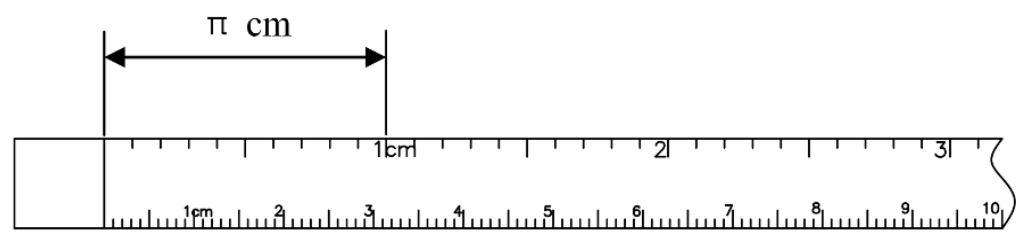

Figure 1. Scale sample of traditional diameter tape

The new barcode diameter tape designed in this paper separates the fractional area from the integer area without a dual scale (Fig. 2).

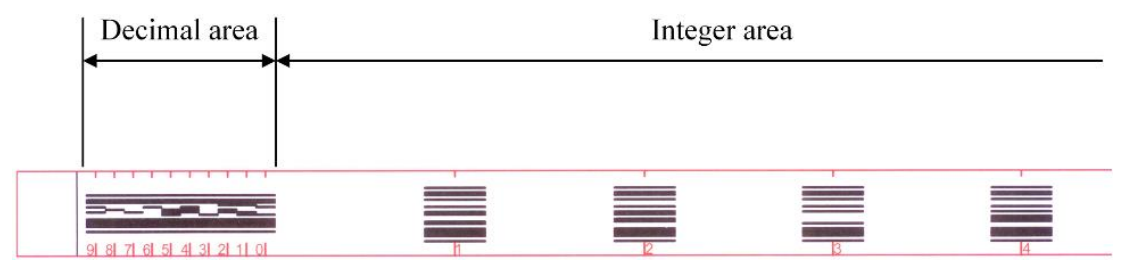

Figure 2. Scale sample of barcode diameter tape

The integer area of the scale interval is $\pi \mathrm{cm}$; the fractional area of the overall length is $\pi \mathrm{cm}$, with ten scales from $0-9$; and the length of the scale interval is $\pi / 10 \mathrm{~cm}$. The measurement principle of conventional diameter tape is shown in Figure 3a; the DBH of the tree is read along the bottom edge of the tape where the left and right ends of the tape cross at the 0-line. The measurement principle of the designed diameter tape is 
shown in Figure $3 b$. The user reads the integer value before zero on the scale and the minimum scale value from the integer value to zero on the scale, which is the sum of the integer value within the fractional area and the fractional value corresponding to the integer scale (Fig. 3).

\section{Principle of barcode encoding}

A barcode can accurately represent numeric and alphabetic data using patterns (black lines and white lines) that can be read rapidly. The diameter tape is a tool used to obtain numeric diameter data, and 1D barcode can represent its corresponding value. However, there is an excessive number of standard patterns of existing barcodes. For example, the classic European Article Number (EAN-13) encoding standard contains 113 patterns. Each pattern is $0.33 \mathrm{~mm}$ in width according to conventional standards. The total width of the barcode is approximately $38 \mathrm{~mm}$, which requires a tape greater than $38 \mathrm{~mm}$ in width. However, the width of conventional diameter tape is approximately $10 \mathrm{~mm}$ and thus does not meet the requirements of existing encoding standards. Therefore, two sets of simple barcodes were designed in this study: a set of black \& white barcodes, and a set of multicolor barcodes.

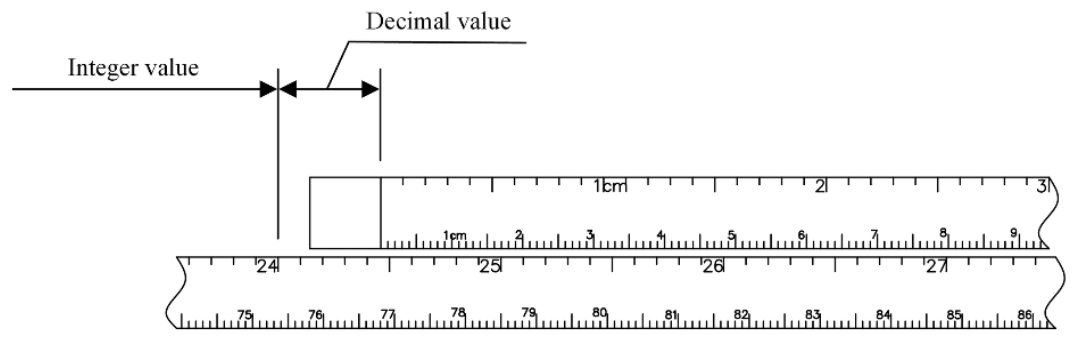

(a)

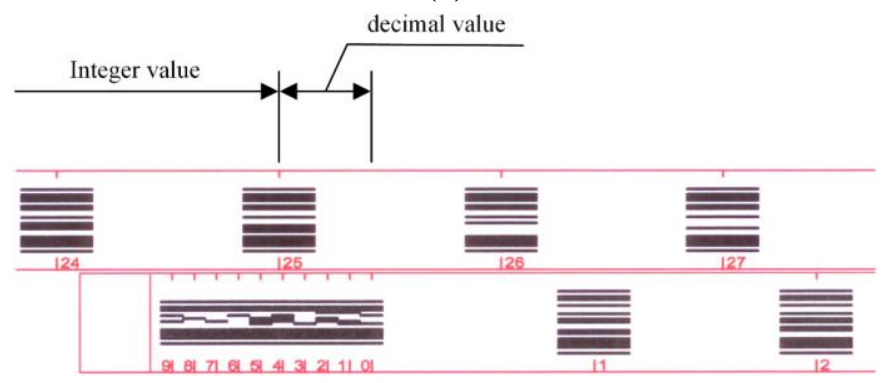

(b)

Figure 3. Comparison of readout principle of diameter tapes

The redesigned barcode consists of Left Quiet Zone, Start Character, Data Character, Stop Character and Right Quiet Zone. The pattern values of each zone except Data Character are shown in Table 1. In the set of black \& white barcodes, the Data Character field refers to the EAN code and uses 7 patterns to represent a digit; the numbers corresponding to the code are shown in Table 2. The integer area requires two digits with 14 patterns, whereas the fractional area requires only one digit with 7 patterns. Different width-encoding modes are used for integer area and fractional area to allow the two parts to be distinguished accurately. The pattern width design is $0.4 \mathrm{~mm}$, so the total tape width is $14 \mathrm{~mm}$. Due to the limited space $(3.14 \mathrm{~mm})$ between scale lines in the fractional area, we made the pattern length $3.14 \mathrm{~mm}$. The results are shown in Figure 2. 
The structure of the multicolor barcodes is identical to that of black \& white barcodes, except that two new colors (red and green) are added, which transforms the binary barcode to a quaternary barcode such that each zone can be represented with fewer patterns. The pattern values are shown in Table 1 and Table 2 . The pattern width design is $0.6 \mathrm{~mm}$; thus, the total width is $9.6 \mathrm{~mm}$. The results are shown in Figure 4.

Table 1. Corresponding zone patterns of black \& white and multicolor barcode

\begin{tabular}{c|c|c|c|c}
\hline \multirow{2}{*}{ Barcode type } & \multicolumn{4}{|c}{ Zone Pattern } \\
& Left Quiet Zone & Start Character & Stop Character & Right Quiet Zone \\
\hline Black \& White & 000000 & 101 & 111101 & 000000 \\
Multicolor & 000 & 10 & 21 & 0000 \\
\hline
\end{tabular}

"0": white; "1": black; "2": red; "3": green

Table 2. Corresponding pattern of data characters

\begin{tabular}{c|c|c}
\hline Digit & \multicolumn{2}{|c}{ Pattern of data character } \\
& Black \&White & Multicolor \\
\hline 0 & 1010010 & 120 \\
1 & 1100110 & 130 \\
2 & 1101100 & 110 \\
3 & 1000010 & 200 \\
4 & 1011100 & 210 \\
5 & 1001110 & 230 \\
6 & 1010000 & 220 \\
7 & 1000100 & 300 \\
8 & 1001000 & 330 \\
9 & 1010100 & 310 \\
\hline
\end{tabular}

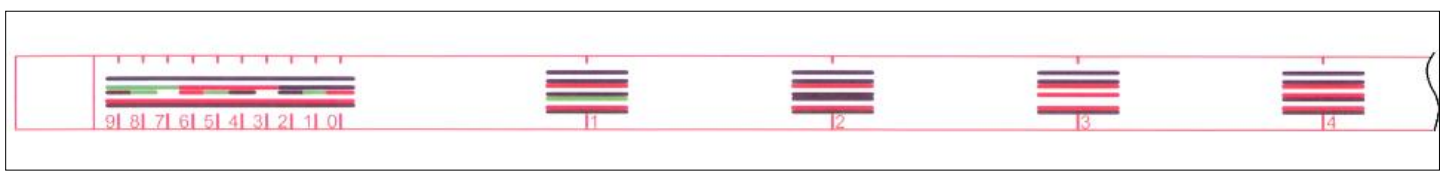

Figure 4. Design of color barcode DBH tape

\section{Principle of barcode decoding}

The redesigned barcode has two parts: an integer area and a fractional area. Neither part follows existing standard code, and there is no ready-made open source library or decoding methods to choose from. In this paper, a new set of decoding schemes was designed to meet the use of barcode diameter tape. The scheme can be used for decoding both black \& white barcode and multicolor barcode. The decoding flowchart is shown in Figure 5.

Typically, a barcode-containing image (Fig. 6) can be captured through a camera-equipped device using the barcode diameter to measure DBH. The color of each pattern in the image can be determined by the values of the three channels of R, G and B. For example, the three-channel values for red are 255, 0, and 0 theoretically. However, the channel values are usually affected by the reflectivity and uniformity of light under natural conditions. In this paper, 3-channel values of the pixels on the mid-perpendicular of the image are counted, and the upper and lower quartiles are obtained. As shown in the 
Table 3, if the pixel's R-channel value is greater than the upper quartile (UQ) of this channel and the G- and B-channel values are less than their respective lower quartiles (LQs), then the pixel color is considered red.

To read the value represented by the barcode, the integer and fractional areas should be found in the image; in Figure 7, this means finding Points (1)-(4). If searching down from right above the image, the pixel color goes through the sequence of "(non-white)-(white)-(non-white \& non-black)-(black)", and the last black pixel is on the topmost module. The width of this pattern can be obtained by continuing to search down, and the midpoint of the module is Point (1). Similarly, Point (4) can be obtained through bottom-up search. However, if the midpoint of Point (1) and Point (4) is adjusted as the starting point for searching Point (2) and Point (3), the product may not conform to this sequence.

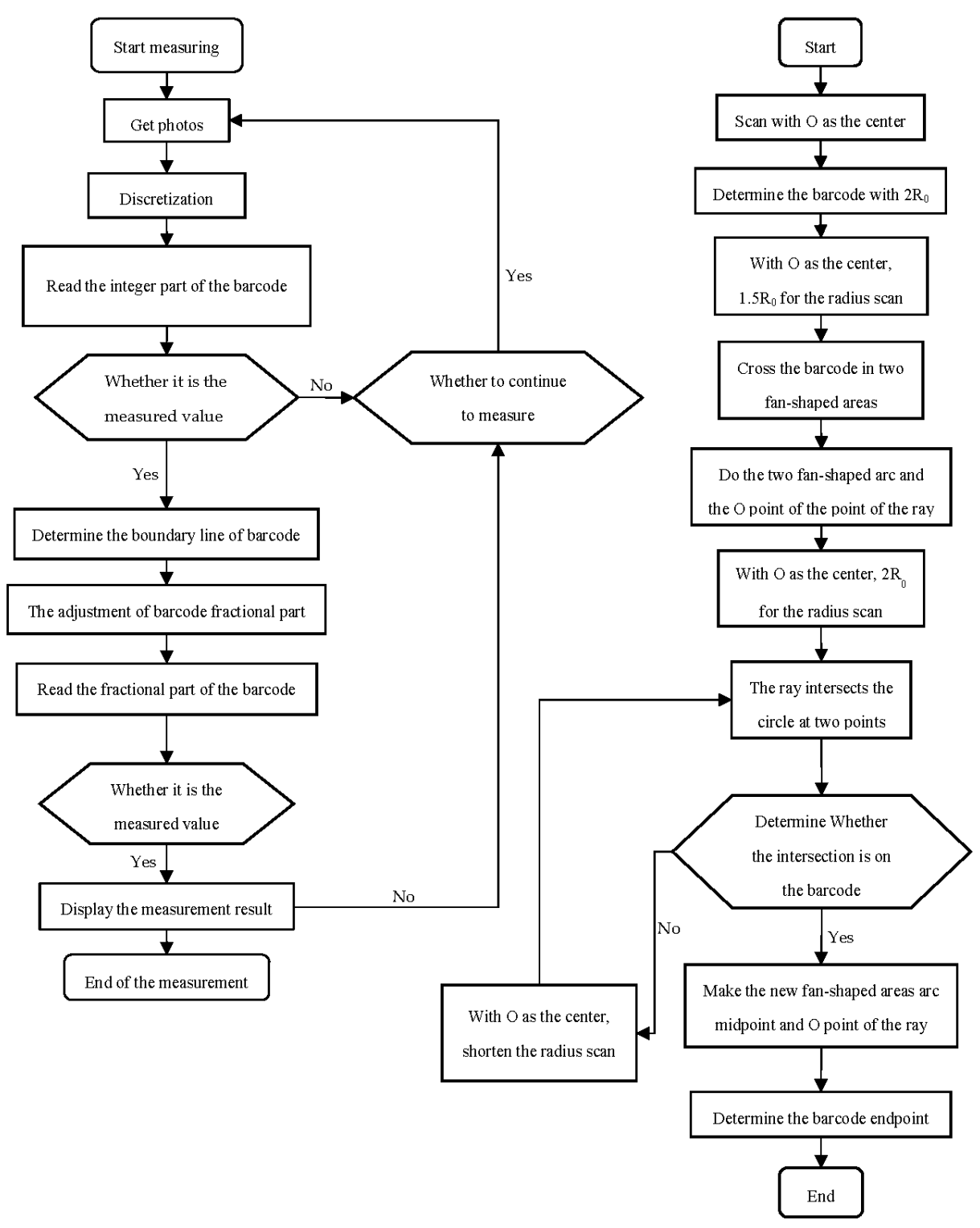

Figure 5. Flowchart of the decoding method 


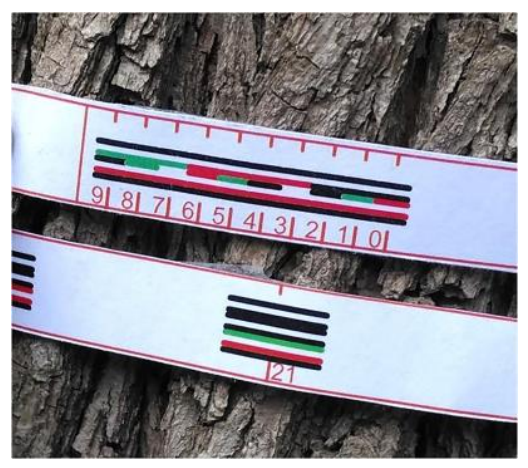

Figure 6. Typical captured barcode-containing image

Table 3. Pixel channel characteristics of different colors

\begin{tabular}{c|c|c|c|c|c|c}
\hline \multirow{2}{*}{ color } & \multicolumn{2}{|c|}{ R-channel value } & \multicolumn{2}{c|}{ G-channel value } & \multicolumn{2}{c}{ B-channel value } \\
& $<$ LQ & $>$ UQ & <LQ & >UQ & <Q & UQ \\
\hline white & - & $\mathrm{Y}$ & - & $\mathrm{Y}$ & - & $\mathrm{Y}$ \\
black & $\mathrm{Y}$ & - & $\mathrm{Y}$ & - & $\mathrm{Y}$ & - \\
red & - & $\mathrm{Y}$ & $\mathrm{Y}$ & - & $\mathrm{Y}$ & - \\
green & $\mathrm{Y}$ & - & - & $\mathrm{Y}$ & $\mathrm{Y}$ & - \\
\hline
\end{tabular}

"LQ": lower quartile; "UQ": upper quartile; "Y": yes

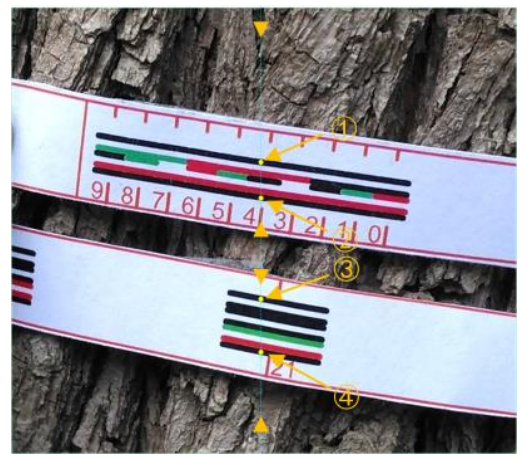

Figure 7. Procedure of edge detection

In this paper, the search starting points are adjusted to the pixel points at the corresponding distance above and below the midpoint. These starting points must be in the gap of tape or in the white area of the tape corresponding to the search direction. The distance is determined by the pattern structures of the barcode diameter tapes, which are shown in Figure 8. For black \& white barcode diameter tape (Fig. 8a), the number of patterns between Point (1) and Point (4) is calculated by Equation 1:

$$
T=(23-0.5+6)+2 g+(9.5+16-0.5)=53.5+2 g
$$

and the distance D should meet the following condition (Equation 2):

$$
\frac{[(23-0.5+6)-T / 2] N}{T}<D<\frac{[T / 2-(23-0.5)] N}{T}
$$


where $\mathrm{N}$ is the number of pixels between Point (1) and Point (4). This interval will be the smallest when $\mathrm{g}=0$, and the range of $\mathrm{D}$ will be as follows (Equation 3):

$$
0.0327 N<D<0.0421 N
$$

Similarly, the smallest interval of multicolor barcode diameter tape is as follows (Equation 4):

$$
0.0319 N<D<0.0532 N
$$

This allows the algorithm to be adapted to both types of tapes, with values in the range of the following (Equation 2):

$$
0.0327 N<D<0.0421 N
$$

After determining the inner patterns and outmost patterns (or Points (1)-(4)), the distance (D12) between Point (1) and Point (2) and the distance (D34) between Point (3) and Point (4) can be calculated, and the wider distance can be considered the integer area. In Figure 7, the area between Point (3) and Point (4) will be seen as the integer area. If the number of patterns in the integer area is $M$, the space between Point (3) and Point (4) is equally divided into M-1 parts, with each equidistant point being the midpoint of each pattern. In this way, the color type of the equidistant points can be read and compared with the code library to obtain the corresponding value.

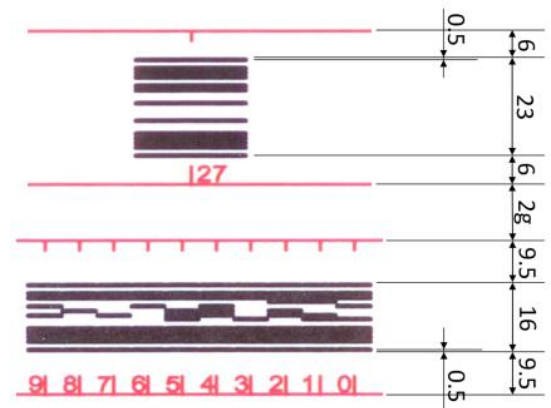

(a)

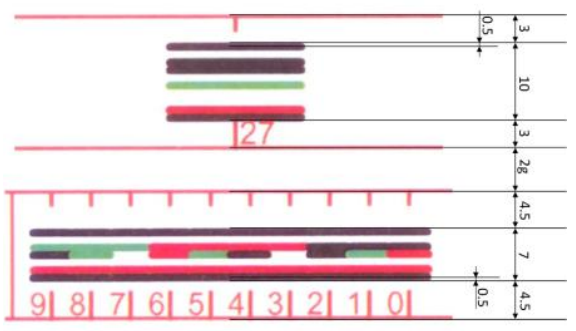

(b)

Figure 8. Pattern distribution of barcodes. The unit is "Patterns", and " 2 " represents the gap width (number of patterns) between the integer area and fractional area of the tape 
The reading of the fractional area is primarily based on the corresponding integer area scale. However, it is difficult to ensure that there is no gap between the integer part and fractional part of tape and that the two parts are parallel during typical measurement. A new algorithm was developed to eliminate these difficulties, and the algorithm diagram is shown in Figure 9. From the figure, we can see that each mid-perpendicular of the pattern in the integer area crosses the corresponding scale line of the integral area, which can determine the scale position of the integer. In this context, the special pattern in the integer area that is closest to the fractional area is chosen as the target for obtaining a mid-perpendicular, and the endpoints of this pattern can be used to calculate the mid-perpendicular. The new algorithm needs to find the endpoints of this pattern; the new algorithm diagram is shown in Figure 9.

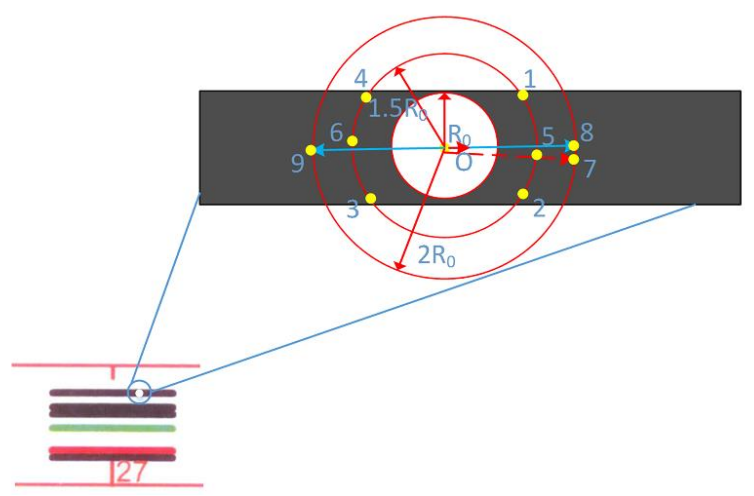

Figure 9. Principle of the decoding algorithm

In this algorithm, a circle, which has its center at Point $\mathrm{O}$ (Point (3) in Figure 7) and a radius of 2 pixels, is determined. If all the pixels in the circle have the same color type as Point $\mathrm{O}$, the radius of the circle is gradually increased until a pixel of a color type different from the color type of Point $\mathrm{O}$ appears in the circle. At the end of this process, the pattern width is $2 R_{0}$ assuming a radius of $R_{0}$. With Point $\mathrm{O}$ as the center and $1.5 R_{0}$ as the radius of a circle, the circle will intersect with the pattern at arc (12)^ and $\operatorname{arc}(34)^{\wedge}$. Point 5 and Point 6 are the midpoints of $\operatorname{arc}(12)^{\wedge}$ and $\operatorname{arc}(34)^{\wedge}$, respectively. With the midpoint as the center and $2 R_{0}$ as the radius of a circle, the ray passing through Point $\mathrm{O}$ and Point 5 intersects with the circle at Point 7 . If Point 7 is in the pattern, it will be corrected to the midpoint 8 of the arc where Point 7 is located. Then, the radius of circle continues to be increased until the intersection of the circle with the corresponding ray is not included in the pattern. The target endpoint should be close to this intersection point and can be obtained by adjusting the intersection point. Similarly, another endpoint can be obtained using this method. These two endpoints are similar to Point A, B in Figure 10, and Point $\mathrm{C}, \mathrm{D}, \mathrm{E}, \mathrm{F}$ can be obtained by the same method. As shown in Figure 10, the mid-perpendicular of Point A, B intersects Line AB at Point P0 and Line CD at Point P1. To amend the error caused by the non-parallel and gap between the integer and fractional parts of the tape, the target fractional code is the code that is intersected by the line passing through Point $\mathrm{P}$ (midpoint of Point P0 and P1) and perpendicular to Line CD or the code between Point S1 and S2. The value can be read in a way similar to that of the integer area. 


\section{Materials and Methods}

\section{The profile of the study sites}

Two plots in Xiangshan $\left(40.00^{\circ} \mathrm{N}, 116.19^{\circ} \mathrm{E}\right)$, located in Beijing, China, were selected for study. A total of 94 standing trees in the plots (each $25 * 25 \mathrm{~m}$ ) were investigated; descriptive statistics of the plots are summarized in Table 4. The tree species in Plot 1 are mainly conifer species, and those in Plot 2 are broad-leaved species. The data in the table were obtained using a caliper. The DBH of each tree was measured by six different observers, and the average values were taken as the true values of the trees.

Since the principles of the black \& white barcode tape and the multicolor barcode tape are the same, the test data were obtained by a multicolor barcode tape. To study the subjectivity of different researchers in measuring $\mathrm{DBH}$ and the effect of observer experience on DBH measurement, the data were measured by six different observers. Numbered patches were attached to the breast height of trees as reference points to ensure that each measurement was collected at the same height.

The reference values were measured using a traditional diameter tape. For comparison with the measurement results obtained by barcode tape, each diameter was measured by the same six different observers.

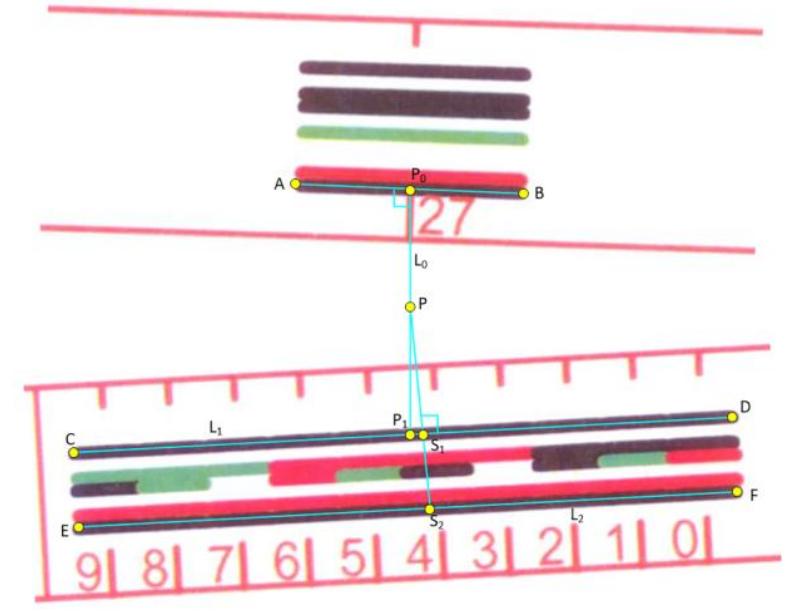

Figure 10. Adjustment and reading of fractional area

Table 4. Summary statistics of DBH in the plots

\begin{tabular}{c|c|c|c|c|c|c}
\hline Plot & Species & N & Mean(cm) & S.D.(cm) & Minimum(cm) & Maximum(cm) \\
\hline \multirow{2}{*}{1} & Platycladus orientalis & 50 & 18.2 & 9.6 & 6.2 & 50.7 \\
& Acer truncatum & 4 & 8.0 & 2.0 & 6.2 & 10.4 \\
\multirow{2}{*}{2} & Catalpa ovata & 37 & 20.6 & 5.6 & 9.5 & 30.0 \\
& Sophora japonicum & 3 & 43.3 & 6.9 & 36.6 & 50.5 \\
\hline
\end{tabular}

\section{Methods}

\section{Error observation}

To estimate the central trend and variability of measurements obtained by different observers using the barcode tape, bias, relative BIAS, root mean squared error (RMSE) 
and relative RMSE were used as evaluation indicators, as defined in (Equation 6) (Equation 9).

$$
\begin{gathered}
\text { Bias }=\frac{1}{n} \sum_{i=1}^{n}\left(d_{i}-d_{r i}\right) \\
\text { Bias\% }=\frac{\text { Bias }}{\bar{d}_{r}} \times 100 \% \\
R M S E=\sqrt{\frac{\sum_{i=0}^{n}\left(d_{i}-d_{r i}\right)^{2}}{n}} \\
R M S E \%=\frac{R M S E}{\overline{d_{r}}} \times 100 \%
\end{gathered}
$$

where $d_{i}$ is the ith measurement, $d_{r i}$ is the ith reference value, $\overline{d_{r}}$ is the mean of references, and $\mathrm{n}$ is the number of measurements.

Generally, the measurements obtained by the traditional diameter tape are widely used as the reference values. Because these values also contain errors and because the purpose of this paper is to compare the accuracy of measurements between a traditional diameter tape and a barcode tape, the measurements obtained by a traditional diameter tape cannot be used as reference values. In this paper, when estimating the bias of the barcode tape measurements, the mean values of observations using the traditional diameter tape by six different observers were taken as the reference values. Furthermore, when comparing the variability of the measurements obtained by the diameter tape and the barcode tape, the means of measurements obtained using the barcode tape by the six different observers were considered as reference values.

\section{Dependence of measurement error}

The purpose of using a barcode tape is to reduce the subjectivity of human-read measurements or the differences in measurements between observers. A linear mixed model can detect differences between groups and is suitable for identifying the error associated with different observers. The model is defined as Equation 10:

$$
\mathrm{y}=X \beta+U \xi+\varepsilon
$$

where $y$ is an $\mathrm{n} \times 1$ observation vector, $\beta$ is the $\mathrm{p} \times 1$ fixed parameters vector, $\mathrm{X}$ is the $\mathrm{n} \times \mathrm{p}$ matrix of the independent variables associated with the fixed parameters, $\xi$ is the $\mathrm{q} \times 1$ intragroup effect parameters vector, $\mathrm{U}$ is the $\mathrm{n} \times \mathrm{q}$ matrix of the explanatory variables associated with the intragroup effect parameters, and $\varepsilon$ is a $\mathrm{n} \times 1$ random error vector.

In this paper, measurement errors were treated as dependent variables, the intercept was the only fixed effect, and observer was considered a random variable. The proportion of errors in the total errors caused by different observers was used as a basis for comparison. 


\section{Results and Discussion}

The measurements of DBH obtained by different observers are shown in Table 5. When the mean measurements observed by the traditional tool were used as the reference values, diameter was on average underestimated by $1.3 \mathrm{~mm}$. However, this bias was not significant $(0.67 \%)$. When the within-group means were used as the reference values, the bias variability $(-1.8 \sim 1.4 \mathrm{~mm})$ of the traditional tool was larger than that of the barcode tape $(-0.7 \sim 0.7 \mathrm{~mm})$. RMSE is an indicator used to assess variability. When the observer used the new measurement tool, regardless of whether the within-group or between-group means were selected as the reference values, the measurement values had less variability $(2.71 \%, 3.92 \%)$ than those obtained with the traditional tool $(4.46 \%)$.

Table 5. Accuracies of the DBH measurements by observers M1-M6 $(n=94$ trees measured by each observer)

\begin{tabular}{c|c|c|c|c|c|c|c|c}
\hline & Observer & Bias (W) & Bias\% (W) & Bias (B) & Bias\% (B) & RMSE (W) & RMSE\% (W) & RMSE (B) \\
\hline & M1 & -1.8 & $-0.88 \%$ & - & - & 13.5 & $6.55 \%$ & - \\
& M2 & -0.1 & $-0.07 \%$ & - & - & 9.8 & $4.74 \%$ & - \\
traditional & M3 & 1.4 & $0.68 \%$ & - & - & 4.4 & $2.14 \%$ & - \\
diameter tape & M4 & -0.1 & $-0.05 \%$ & - & - & 14.3 & $6.96 \%$ & - \\
& M5 & 0.2 & $0.12 \%$ & - & - & 8.5 & $4.14 \%$ & - \\
& M6 & 0.5 & $0.23 \%$ & - & - & 4.6 & $2.23 \%$ & - \\
& Mean & 0.0 & $0.00 \%$ & - & - & 9.2 & $4.46 \%$ & - \\
\hline & M1 & -0.7 & $-0.32 \%$ & -1.0 & $-0.50 \%$ & 4.2 & $2.03 \%$ & 5.6 \\
& M2 & 0.1 & $0.07 \%$ & -0.2 & $-0.11 \%$ & 5.6 & $2.73 \%$ & 7.7 \\
& M3 & -0.1 & $-0.07 \%$ & -0.5 & $-0.25 \%$ & 4.1 & $2.01 \%$ & 3.9 \\
& M4 & 0.7 & $0.34 \%$ & 0.3 & $0.16 \%$ & 7.2 & $3.50 \%$ & 10.9 \\
& M5 & -0.5 & $-0.24 \%$ & 9.5 & $4.86 \%$ & 9.0 & $4.37 \%$ & 13.3 \\
& M6 & 0.1 & $0.04 \%$ & -0.3 & $-0.14 \%$ & 3.3 & $1.63 \%$ & 6.3 \\
& Mean & -0.1 & $-0.03 \%$ & 1.3 & $0.67 \%$ & 5.6 & $2.71 \%$ & 7.9 \\
\hline
\end{tabular}

"W" means the within-group evaluations; "B" means the between-group evaluations; M1-M6 means different observers

Regarding the variability of measurements obtained with the diameter tape, $13.7 \%$ was due to differences among observers. When using the barcode tape, the corresponding value was $0 \%$. However, if the means of measurements obtained with the traditional tape were selected as the reference values, the corresponding value was $21.0 \%$. This larger variability was due to the differences between the mean values of different measurement tools; thus, the barcode tape was unable to eliminate the differences between observers. However, this new tool can reduce this difference. To determine the reduction in this difference, many comparative tests should be conducted over the long term.

The measurement results are expected to be better than the results from the remote sensing, such as relascope, TLS, MLS and camera. Kalliovirta et al. (2005) evaluated the availability of laser-relascope in forest inventory and reported an $8.2 \mathrm{~mm}$ standard error of DBH estimations, i.e. $4.7 \%$ from the mean diameter; obviously, the results have greater variability compared to our results due to our new tool using a contact measurement method. Vastaranta et al. (2009) evaluated the accuracy of the laser-camera in estimating the DBH of the tree; their results showed the standard errors 
of DBH measurements were $8.3 \mathrm{~mm}$ and $8.5 \mathrm{~mm}$. Although the device used a method of automatically estimating the DBHs to avoid subjective interference, it is less accurate than our tool because it is also a remote sensing measurement tool. Miller et al. (2015) used the digital photogrammetry to obtain point clouds to estimate the DBHs of the trees; the RMSE of the DBH estimation of individual trees was $2.39 \mathrm{~cm}$. This approach avoided subjective interference and improved work efficiency, but its accuracy is still lower than our tool due to limited camera resolution and processing methods. Obviously, the accuracy of the barcode DBH tape data shows that it has the potential to measure DBH accurately. The results in this study indicated that the barcode DBH tape is an attractive and accurate technology for DBH measurements, which may be applied as the references to former remote sensing methods.

\section{Conclusions}

In this study, two barcode diameter tapes (black \& white and multicolor barcode tapes) were designed. A new decoding algorithm was designed and developed as an application that was installed on a mobile phone with a wide-angle camera for parsing and recording the measurements of these new diameter tapes. The system was tested under typical forest conditions. When the mean values obtained with a traditional diameter tape were selected as reference values, the bias of the measurements was not significant $(1.3 \mathrm{~mm})$. The RMSE of the DBH was $5.6 \mathrm{~mm}$. In addition, the DBM measurements obtained with a traditional tape were biased; specifically, they tended to be overestimates. The results also showed that the new tool could reduce the subjectivity of observers when reading observations and potentially replace traditional diameter tapes.

In the future, many comparative trials should be conducted to determine the precise amount of subjective improvement attained with the barcode tape. These tests should be conducted under different forest conditions, such as different canopy closures, ages, and forest types. In addition, there are some problems with the algorithm that cannot be completely solved. For example, when the angle between the integer tape and decimal tape is too large, the decimal value may not be accurately determined. This problem should be assessed quantitatively and appropriately resolved.

\section{REFERENCES}

[1] Bijak, S., Sarzyński, J. (2015): Accuracy of smartphone applications in the field measurements of tree height. - Folia Forestalia Polonica 57.

[2] Drexhage, M., Colin, F. (2001): Estimating root system biomass from breast-height diameters. - Forestry 74: 491-497.

[3] Gering, L. R., May, D. M. (1995): The Relationship of Diameter at Breast Height and Crown Diameter for Four Species Groups in Hardin County, Tennessee. - Southern Journal of Applied Forestry 19: 177-181.

[4] Juujarvi, J., Heikkonen, J., Brandt, S. S., Lampinen, J. (1998): In Digital-image-based tree measurement for forest inventory, Intelligent Robots and Computer Vision XVII: Algorithms, Techniques, and Active Vision. - International Society for Optics and Photonics: 114-124.

[5] Kalliovirta, J., Laasasenaho, J., Kangas, A. (2005): Evaluation of the Laser-relascope. Forest Ecology and Management 204: 181-194. 
[6] Liang, X., Hyyppä, J., Kaartinen, H., Holopainen, M., Melkas, T. (2012): Detecting Changes in Forest Structure over Time with Bi-Temporal Terrestrial Laser Scanning Data. - ISPRS International Journal of Geo-Information 1: 242-255.

[7] Liang, X., Hyyppä, J., Kukko, A., Kaartinen, H., Jaakkola, A., Yu, X. (2014a): The Use of a Mobile Laser Scanning System for Mapping Large Forest Plots. - Geoscience and Remote Sensing Letters, IEEE 11: 1504-1508.

[8] Liang, X., Jaakkola, A., Wang, Y., Hyyppä, J., Honkavaara, E., Liu, J., Kaartinen, H. (2014b): The Use of a Hand-Held Camera for Individual Tree 3D Mapping in Forest Sample Plots. - Remote Sensing 6: 6587-6603.

[9] Maas, H. G., Bienert, A., Scheller, S., Keane, E. (2008): Automatic forest inventory parameter determination from terrestrial laser scanner data. - International Journal of Remote Sensing 29: 1579-1593.

[10] Maia Araujo, T., Higuchi, N., Carvalho, J. (1999): Comparison of formulae for biomass content determination in a tropical rain forest site in the state of Para Â, Brazil. - Forest Ecology and Management 117: 43-52.

[11] Melkas, T., Vastaranta, M., Holopainen, M. (2008): Accuracy and efficiency of the Laser-camera. - SilviLaser: 17-19.

[12] Miller, J., Morgenroth, J., Gomez, C. (2015): 3D modelling of individual trees using a handheld camera: Accuracy of height, diameter and volume estimates. - Urban Forestry \& Urban Greening 14: 932-940.

[13] Molinier, M., Lopez-Sanchez, C., Toivanen, T., Korpela, I., Corral-Rivas, J. J., Tergujeff, R., Häme, T. (2016): Relasphone-Mobile and Participative In Situ Forest Biomass Measurements Supporting Satellite Image Mapping. - Remote Sensing 8: 869-891.

[14] Olofsson, K., Olsson, H. (2017): Estimating tree stem density and diameter distribution in single scan terrestrial laser measurements of field plots: a simulation study. Scandinavian Journal of Forest Research 33: 1-20.

[15] Ouimet, R., Camiré, C., Brazeau, M., Moore, J.-D. (2008): Estimation of coarse root biomass and nutrient content for sugar maple, jack pine, and black spruce using stem diameter at breast height. - Canadian Journal of Forest Research 38: 92-100.

[16] Popescu, S., Wynne, R., Nelson, R. (2003): Measuring individual tree crown diameter with lidar and assessing its influence on estimating forest volume and biomass. Canadian Journal of Remote Sensing 29: 564-577.

[17] Qu, Y., Wang, J., Song, J., Wang, J. (2017): Potential and Limits of Retrieving Conifer Leaf Area Index Using Smartphone-Based Method. - Forests 8: 217.

[18] Riaño, D., Meier, E., Allgöwer, B., Chuvieco, E., Ustin, S. (2003): Modeling airborne laser scanning data for the spatial generation of critical forest parameters in fire behavior modeling. - Remote Sensing of Environment 86: 177-186.

[19] Saarinen, N., Vastaranta, M., Vaaja, M., Lotsari, E., Jaakkola, A., Kukko, A., Kaartinen, H., Holopainen, M., Hyyppä, H., Alho, P. (2013): Area-Based Approach for Mapping and Monitoring Riverine Vegetation Using Mobile Laser Scanning. - Remote Sensing 5: 5285-5303.

[20] Srinivasan, S., Popescu, S., Eriksson, M., Sheridan, R., Ku, N.-W. (2015): Terrestrial Laser Scanning as an Effective Tool to Retrieve Tree Level Height, Crown Width, and Stem Diameter. - Remote Sensing Letters 7: 1877-1896.

[21] Sumida, A., Miyaura, T., Torii, H. (2013): Relationships of tree height and diameter at breast height revisited: Analyses of stem growth using 20-year data of an even-aged Chamaecyparis obtusa stand. - Tree physiology 33.

[22] Varjo, J., Henttonen, H., Lappi, J., Heikkonen, J., Juujärvi, J. (2019): Digital horizontal tree measurements for forest inventory.

[23] Vastaranta, M., Latorre, E. G., Luoma, V., Saarinen, N., Holopainen, M., Hyyppä, J. (2015): Evaluation of a Smartphone App for Forest Sample Plot Measurements. - Forests 6: 1179-1194. 
[24] Villasante, A., Fernandez, C. (2014): Measurement errors in the use of smartphones as low- cost forestry hypsometers. - Silva Fennica 48: 11.

[25] Watt, P., Donoghue, D. (2005): Measuring forest structure with terrestrial laser scanning. - International Journal of Remote Sensing 26.

[26] Wieser, M., Mandlburger, G., Hollaus, M., Otepka, J., Glira, P., Pfeifer, N. (2017): A Case Study of UAS Borne Laser Scanning for Measurement of Tree Stem Diameter. Remote Sensing 9: 1154.

[27] Yu, X., Liang, X., Hyyppä, J., Kankare, V., Vastaranta, M., Holopainen, M. (2013): Stem biomass estimation based on stem reconstruction from terrestrial laser scanning point clouds. - Remote Sensing Letters 4: 344-353. 